\title{
THE RELATIONSHIP BETWEEN MORPHOLOGICAL INDICATORS OF HUMAN BODY AND POSTURE
}

\author{
Radka Molikova ${ }^{\mathrm{a}}$, Marcela Bezdickova ${ }^{\mathrm{a}}$, Katerina Langova ${ }^{\mathrm{b}}$, Vladimir Holibka ${ }^{\mathrm{a}}$, \\ Ondrej David ${ }^{\mathrm{a}}$, Zdenka Michalikovac ${ }^{\mathrm{c}}$ Jarmila Rehorova $^{\mathrm{d}}$
}

\author{
a Department of Anatomy, Faculty of Medicine and Dentistry, Palacky University Olomouc, Czech Republic \\ ${ }^{b}$ Department of Medical Biophysics, Faculty of Medicine and Dentistry, Palacky University Olomouc, Czech Republic \\ c Graphic Designer, Faculty of Medicine and Dentistry, Palacky University Olomouc, Czech Republic \\ d Department of Head Nurse, University Hospital Olomouc, Czech Republic \\ e-mail:molikovaradka@yahoo.co.uk
}

Received: May 30, 2006; Accapted: June 15, 2006

Key words: Posture/Postural stability/Weight Bearing Squat/Body position/Morphological indicators/NeuroCom Balance

Background: Posture and Stability Control is a process of maintaining balance and position of the body and its parts in a constantly changing environment. It is an important regulatory mechanism of the body, because it precedes movement, is upon the completion of which this system tries to maintain the body position.

Aim: The purpose of this long term study is to determinate by special testing which morphological indicators and how influence postural stability and its control.

Method: This study used testing with NeuroCom Balance-12 protocols, anthropometric measuring - 32 values, anamnestic questionnaire. For this article we analyzed one chosen protocol: Weight Bearing Squat Test.

Results: In this research, 41 individuals took part in it. 22 (53.66 \%) were female, $19(46.34 \%)$ were male. Interesting statistically significant coherences were discovered during analysis of Weight Bearing Squat protocol on level $\mathrm{p}<0.01, \mathrm{p}<0.05$ and the Student's two-optional T-test proved statistically significantly higher values of Left Body Weight erect position and Left Body Weight $30^{\circ}$ knee flexion in men in comparison with women.

Conclusions: This study shows us a direction and we may consider it a convenient base for more specific continuation in this problematic area.

\section{INTRODUCTION}

Observing and recognizing the patient's walk or analyzing postural stability may be the most important part of the neurological examination. Seeing how the patient initiates a planned action, evaluating the ability to maintain balance while "repetitively hurling oneself into space" - as the act of walking has been described, and analyzing an abnormal gait for clues to the nature of the deficit, all provide valuable information ${ }^{5,6,21}$. The scientific question was: To what extent individual morphological signs influence postural stability and its control?

The study of postural dynamics is important not only to understand disorders of impaired equilibrium and protective reactions, but also to the design prothesis and functional neuromuscular stimulation as aids to patients with impaired postural stability and locomotion ${ }^{12}$.

Measurement of balance has numerous potencial applications in medical training, such as determining the effects of injury, surgery, and external devices ${ }^{18}$.

\section{MATERIALS AND METHODS}

The tested individuals (regardless their age) underwent examination that was divided into 3 stages: step 1 - measuring of morphological indicators, step 2 - testing with NeuroCom Balance, and step 3 - anamnestic questionnaire. This research is intended as a long term study, unprecedented to date. All probands were duly educated about the process of examination and they signed an informed consent. The probands criteria were no current or serious past medical diagnosis, no medication affecting the CNS or balance control, no symptoms of dizziness of vestibular or neurologic disorders, no psychological disorders, normal vision, no unexplained medical history within the last 6 months.

\section{Measuring of morphological indicators}

Measuring of morphological indicators was performed under standard conditions on the body of minimally dressed proband. We measured 32 anthropometric figures in every individual under standard conditions using specified way and instruments. As measured figures, we chose standardized data according to Martin-Saller and Nelson ${ }^{2,13,17}$. During measurement, we kept to the precisely defined anthropometric points ${ }^{15,17}$. These points on 
a body represent the same named points on the skeleton and it is, together with the use of anthropometric figures, possible to count and define body indices.

\section{Testing with neurocom balance}

The second stage was the measurement of postural stability on NeuroCom Balance machine, by NeuroCom International, which is located in Kinesiologic laboratory in Clinic of Exercise and Sports Medicine of Teaching Hospital Olomouc. NeuroCom International, Inc specializes in the development of computerized tools for the neuro-assessment and physiotherapy of patients with balance and mobility disorders. 12 protocols were selected from a broad spectrum and each parameter was measured as a three-trial average score.

\section{Anamnestic questionnaire}

The personal anamnesis was taken on the basis of completing a questionnaire related to our research: sex, age, laterality, sports activity, absence of inborn or acquired body defects, serious illness or injury, which would impact or distort the research results ${ }^{8,19}$. Other information regarding eye defects, dizziness and the status of medication which would otherwise influence the body stability were also ascertained. We strictly adhered by personal data security.

Tab. 1. Average characteristics of the basic group.

\begin{tabular}{|l|c|c|c|c|c|}
\hline & $\Sigma$ & Age & $\begin{array}{c}\text { Height/ } \\
\mathrm{cm}\end{array}$ & $\begin{array}{c}\text { Weight/ } \\
\mathrm{kg}\end{array}$ & BMI \\
\hline $\begin{array}{l}\text { Complete } \\
\text { group }\end{array}$ & 41 & 30.10 & 172.80 & 70.60 & $\begin{array}{c}23.46 / \\
\text { NORMAL }\end{array}$ \\
\hline Female & 22 & 31.41 & 165.80 & 63.40 & $\begin{array}{c}23.12 / \\
\text { NORMAL }\end{array}$ \\
\hline Male & 19 & 28.58 & 180.90 & 79.10 & $\begin{array}{c}23.85 / \\
\text { NORMAL }\end{array}$ \\
\hline
\end{tabular}

In this research, 41 individuals took part. 22 (53.66\%) were female, 19 (46.34 \%) were male. Personal anamnesis showed that no one from the group of probands participating in the research, had at the time of measurement, any health problems and did not suffer from any illness or physical limitations which would negatively influence the body posture. 11030 data of postural stability and 1312 data from anthropometric measuring were enlisted during the research. Because of the scope of the research and the number of data acquired, we only opted for comparison of morphological indicators: height $[\mathrm{cm}]$, weight $[\mathrm{kg}], \mathrm{BMI}^{20}$, blood pressure systolic/diastolic [ $\mathrm{mm} \mathrm{Hg}$ ], circumference of chest in normal position maximal $[\mathrm{cm}]$, circumference of chest in normal position minimal [cm], circumference of abdomen [cm], circumference of waist [cm], circumference of gluteus [cm], length of upper limb [cm], length of lower limb [cm], length of foot [cm], width of foot type I. [cm] and II [cm]. Within these morphological indicators, we analyzed chosen protocol, which is Weight Bearing Squat (WBS). The basis group characteristic is shown in table 1 .

\section{Weight bearing squat}

During the Weight Bearing Squat (WBS) assessment, the patient is instructed to maintain equal weight on the two legs while standing erect and then squatting in three positions of knee flexion. The percentage of body weight borne by each leg (Left and Right Body Weight) is measured with the patient standing at $0^{\circ}$ (erect position), $30^{\circ}$, $60^{\circ}$, and $90^{\circ}$ of knee flexion. In the erect position, most body weight is borne through the skeletal system, and relatively less stress is placed on the knee and hip joints ${ }^{22}$.

\section{RESULTS}

In the comparison of morphological indicators of our group of probands with the statistical analysis, selected morphological indicators and selected tests on Neurocom

Tab. 2. Description of coherences between the Weight Bearing Squat Test (WBS) and the proband's sex.

\begin{tabular}{|l|c|c|c|c|c|c|c|}
\hline & \multicolumn{2}{|c|}{$\begin{array}{c}\text { Levene's Test for } \\
\text { Equality of Variances }\end{array}$} & \multicolumn{4}{c|}{ t-test for Equality of Means } \\
\cline { 2 - 8 } & F & Sig. & $\mathrm{t}$ & df & $\begin{array}{c}\text { Sig. } \\
\text { (2-tailed) }\end{array}$ & $\begin{array}{c}\text { Mean Difference } \\
\text { Std. Error } \\
\text { Difference }\end{array}$ \\
\hline L_\%B_WT_0 & 2.402 & 0.129 & 0.000 & 38 & 1.000 & 0.00 & 2.96 \\
\hline L_\%B_WT_3 & 0.212 & 0.648 & 3.111 & 38 & 0.004 & 3.33 & 1.07 \\
\hline L_\%B_WT_6 & 3.692 & 0.062 & 2.483 & 38 & 0.018 & 3.34 & 1.35 \\
\hline L_\%B_WT_9 & 0.004 & 0.952 & 0.557 & 38 & 0.581 & 0.78 & 1.41 \\
\hline R_\%B_WT_0 & 2.346 & 0.134 & -2.016 & 37 & 0.051 & -2.78 & 1.38 \\
\hline R_\%B_WT_3 & 0.212 & 0.648 & -3.111 & 38 & 0.004 & -3.33 & 1.07 \\
\hline R_\%B_WT_6 & 3.692 & 0.062 & -2.483 & 38 & 0.018 & -3.34 & 1.35 \\
\hline R_\%B_WT_9 & 0.004 & 0.952 & -0.557 & 38 & 0.581 & -0.78 & 1.41 \\
\hline
\end{tabular}




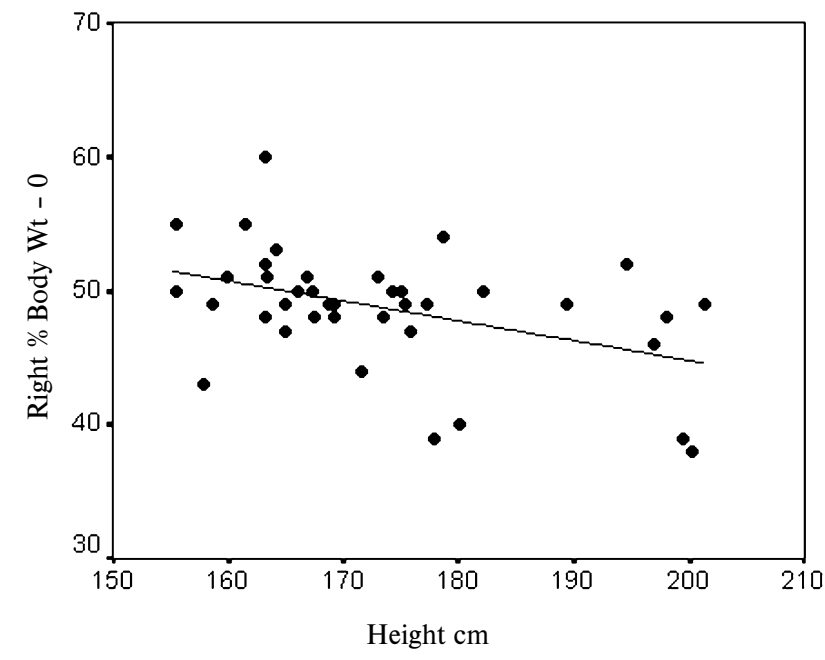

Chart 1. Description of correlation between Right Body Weight $0^{\circ}$ and Height $-0.439(\mathrm{p}<0.01)$.

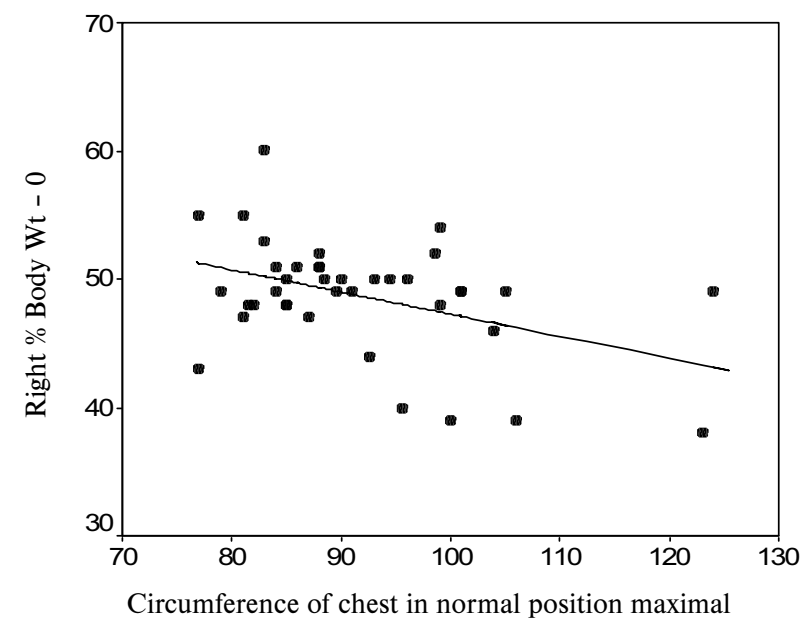

Chart 3. Description of correlation between Right Body Weight $0^{\circ}$ and circumference of chest in normal position maximal $-0.423(\mathrm{p}<0.01)$.

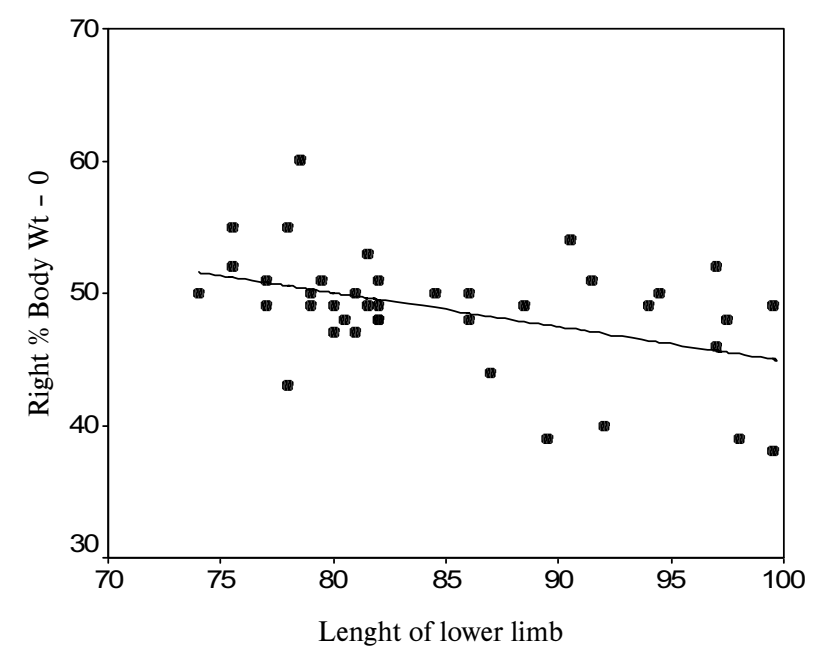

Chart 5. Description of correlation between Right Body Weight $0^{\circ}$ and Length of lower limb -0.439 $(\mathrm{p}<0.01)$.

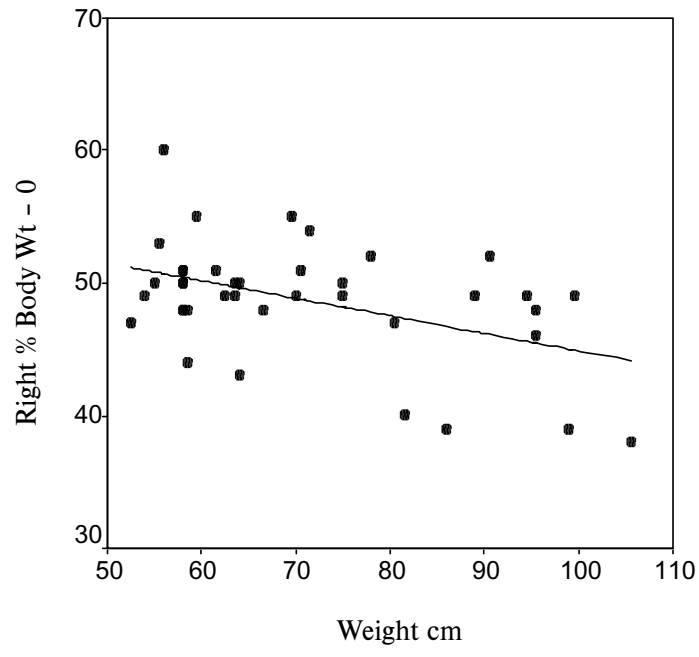

Chart 2. Description of describe correlation between Right Body Weight $0^{\circ}$ and Weight -0.455 $(\mathrm{p}<0.01)$.

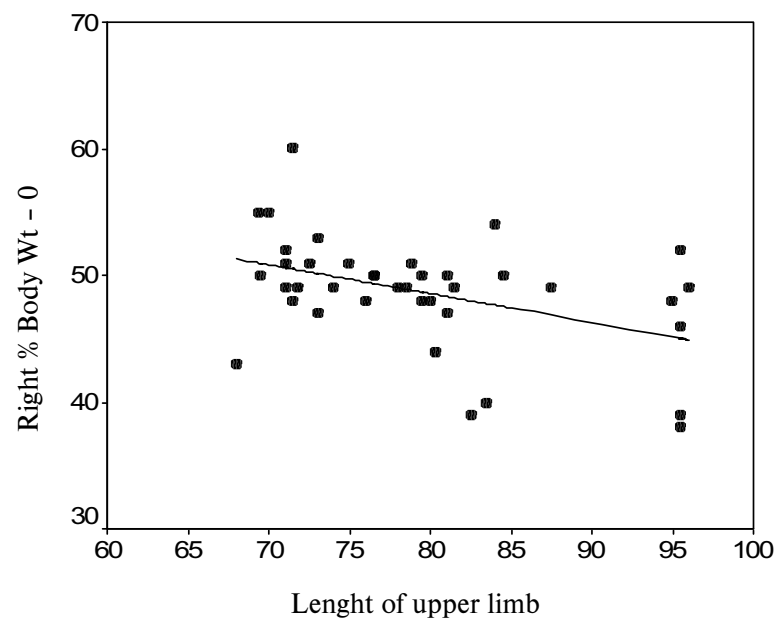

Chart 4. Description of correlation between Right Body Weight $0^{\circ}$ and length of upper limb -0.431 $(\mathrm{p}<0.01)$. 
Balance, we have discovered coherences between the Weight Bearing Squat Test and the proband's sex, by the means of Student's two-optional T-test (tab. 2) and statistically significant coherences by the means of Pearcon's correlation.

The Student's two-optional T-test proved statistically significantly higher values of Left Body Weight $0^{\circ}$ - erect position (L \% B WT 0) and Left Body Weight $30^{\circ}$ knee flexion (L \% B WT 3) in men in comparison with women and statistically significantly lower values of Right Body Weight $0^{\circ}$ - erect position (R \% B WT 0) and Right Body Weight $30^{\circ}$ knee flexion (R \% B WT 3) in men in comparison with women. The situation is illustrated in the table 2 .

In WBS analysis we discovered a significant statistical relations, mainly concerning in erect position Right Body Weight $0^{\circ}$ (R \% B WT 0) and proband's height $0.437(p<0.01)$, weight $-0.455(p<0.01)$, circumference of chest $-0.423(p<0.01)$, length of upper limb -0.431 $(p<0.01)$, length of lower limb $-0.439(p<0.01)$. The situation is illustrated in the chart 1-5.

Other significant relations were discovered between Left Body Weight $0^{\circ}$ (L \% B WT 0) and proband's height 0.359 ( $p<0.05$ ), Left Body Weight $60^{\circ}$ (R \% B WT 6) and length of upper limb 0.313 ( $\mathrm{p}<0.05$ ), Left Body Weight $90^{\circ}$ (R \% B WT 9) and blood pressure systolic -0.373 ( $\mathrm{p}<0.05$ ), Right Body Weight $0^{\circ}$ ( R \% B WT 0) and circumference of chest in normal position minimal -0.401 $(p<0.05)$, length of foot $-0.384(p<0.05)$, width of foot type I. 0.402 ( $p<0.05)$, width of foot type II. 0.380 ( $\mathrm{p}<0.05$ ), Right Body Weight $60^{\circ}$ (R \% B WT 6) and length of upper limb $-0.313(\mathrm{p}<0.05)$.

\section{DISCUSSION}

The aim of this research was to discover, if the individual morphological characteristic can influence postural stability, and if so, which of these characters are significant and how much they contribute to influencing postural stability. In current medical research, many studies pursue research of postural stability, from a perspective of neurology, physiotherapy and orthopaedics, by means of computerised and standardized non-digital methods. Measurement of postural control is difficult, because the location of the centre of the gravity is not determined easily ${ }^{10}$. Measurement of postural control is also difficult because, although an infinite number of equilibrium position exist for the human body, many of them, including an erect, bipedal stance, allow only a few degrees of body sway $^{9,10}$. We can state that a study of such scope and direction has so far not been published.

P. Allard's group ${ }^{1}$ was dealing with the same question and presented that somatotypes should be considered when assessing standing posture in both healthy subjects and patients. The Allard's study presented an important guide-post for us.

We are able to presume from initial results of our study that statistically significant correlation between balance control and selected morphological indicators was proven most distinctly in WBS analysis that the percentage of bodyweight borne in erect position on the right leg at the group of our probands statistically significantly correlates $(\mathrm{p}<0.01)$ with their height, weight, circumference of chest in normal position maximal and length of upper limb, length of lower limb, furthermore with $(p<0.05)$ length of foot, width of foot I., width of foot II., which are body structures that participate in maintaining balance in an erect position.

The relation between the length of upper limb and Right Body Weight in erect position and when standing at $60^{\circ}$ of knee flexion remains an interesting finding, and the relation between length of upper limb and Left Body Weight, when standing at $60^{\circ}$ of knee flexion is statistically significant.

In analyses of other tests, such as SOT, we found that Sensory Organization Test Equilibrium, where we can state that with increasing height of proband, there is a better control over balance under conditions Eyes open, sway referenced surface and visual surround, same relation applies concerning the width of proband's foot.

According to Colledge's study ${ }^{4}$ all the groups were more dependent on proprioception than vision in the maintance of balance, but when reliable pressoreceptor information was removed, dependence on vision increased $^{4,11,14}$ and the relative contributions of the sensory system to balance did not alter with advancing age. Kamen's study found in the study about the develop an inexpective, efficient system for clinical assessment of static and dynamic balance that the instrumentation can be used to discriminate among balance tasks and to differentiate healthy older adults from those with a tendency for frequent falls ${ }^{14}$.

Many studies pursue testing and analysis of walk itself, in relation to neurological examination and fall prevention. Lamb et $\mathrm{al}^{16}$ found that fall gait speed and transfer ability were related to falls in a bivariate analysis but that only transfer ability was related to fall in a multivariate analysis. Hyndman et $\mathrm{al}^{11}$ found that mobility was not a factor in fall events. One of the reasons that Lamb et al found such a relationship but Hyndman et al did not may have been the method in which mobility was measured. Lamb et al used measure of walking (i.e., gait, speed) along with specific one-item functional test such as rising from a chair, transfers, and bed mobility, whereas Hyndman et al used only one functional measure (Rivermead Mobility Index). Taken separately, these one-item measures are related to fall incidence, but once they are combined with other variables that may be related to fall (e.g., depression, balance, ADL ability), their impact is diminished or negated. This may suggest the importance of comprehensive assessments that focus on the physical, cognitive-psychological, and environmental factors ${ }^{11,16}$.

We hope that by a complete analysis of data, which is a part of the thesis, we shall gain data evidencing the relation between morphological indicators and walk.

The importance of this research is not only in a meaningful contribution towards care of patients with neu- 
rodegenerative disorders and stroke, i.e. mainly in the prevention of possible falls as stated by Harris et a ${ }^{7}$, but also for the medical staff from the perspective of working environment ergonomics. Balance retraining is a major component of rehabilitation programs for patients with neurological disorders, musculoskeletal impairments, and elderly people with balance deficits ${ }^{3}$.

\section{LIST OF ABBREVIATIONS}

CNS Central Nervous System

COG Centre of Gravity

BMI Body Mass Index

kg kilogram

cm centimetre

mm millimetre

Hg mercury

tab. table

Fig. figure

WBS Weight Bearing Squat

(L \% B WT 0) Left Body Weight $0^{\circ}$ erect position

(L \% B WT 3) Left Body Weight $30^{\circ}$ knee flexion

(L \% B WT 6) Left Body Weight $60^{\circ}$ knee flexion

(L \% B WT 9) Left Body Weight $90^{\circ}$ knee flexion

(R \% B WT 0) Right Body Weight $0^{\circ}$ erect position

(R \% B WT 3) Right Body Weight $30^{\circ}$ knee flexion

(R \% B WT 6) Right Body Weight $60^{\circ}$ knee flexion

(R \% B WT 9) Right Body Weight $90^{\circ}$ knee flexion

SOT-E Sensory Organization Test-Equilibrium

\section{REFERENCES}

1. Allard P, Nault ML, Hinse S, Leblanc R, Labelle H. Relationship between morphology somatotypes and standing posture equilibrium. Amn. Hum. Biol. 28:624-633 [source on the internet]. 2001 [cited 2006 Aug 22]. Available from: http:// www. ncbi.nml.nih. gov

2. Benes J. Anthropology I. Prague: $1^{\text {st }}$ edit State education publishing. ISBN 80-210-0116-X, 1990.

3. Brouwer B, Culham GE, Liston RA, Grant T. Normal variability of postural measures: Implications for the reliability of relative balance performance outcomes. Scand. J. Rehab. Med. 1998; 30:131- 137 .
4. Colledge NR, Cantley P, Peaston I, Brash H, Lewis S, Wilson JA. Ageing and Balance: The measurement of Spontaneous Sway by posturography. Geronthology 1994; 40:273-278.

5. Greenberg D, Aminof M, Simon R. Clinical neurology. McGrawHill $5^{\text {th }}$ edition. ISBN 7-117-4950-2, 2002.

6. Greenstein B, Greenstein A. Color Atlas of Neuroscience: Neuroanatomy and Neurophysiology. Thieme Sttutgart SRN, ISBN 0-086577-710-1, 2000.

7. Harris JE, et al. Relationship of Balance and Mobility to Fall Incidence in people with Chronic Stroke. Physical Therapy 2005; 85:150-157.

8. Hausman KA, Hartshorn MN, Evans JA. Analyzing Neurological Status: Adult Assessment. Baltimore: Resource Application Inc. USA, ISBN 0-932491-24-3, 1985.

9. Hayes KC. Biomechanics of posture control. Exerc. Sport. Sci. Rev. 1982; 10:363-391.

10. Horak BF. Clinical Measurement of Postural Control in Adult. Physical Therapy 1987; 67:1881-5.

11. Hyndman D, Ashburn A, Stack E. Fall events among people with stroke living in the community: circumstances of falls and characteristics of fallers. Arch. Phys. Med. Rehabilitation 2002; 83:165-170.

12. Johansson R, Magnusson M. Human postural dynamics. Crit. Rev. Biomed.Eng. 1991; 18(6):413-437.

13. Jurmain R, Nelson H, Kilgore L, Trevathan W. Introduction to Physical Anthropology. USA: 8th Edition Wadsworth, ISBN 0534-51444-8; 2000.

14. Kamen G, Patten C, Du DC, Sison S. An Accelerometry-Based System for Assessment of Balance and Postural Sway. Gerontology 1998; 44:40-45.

15. Klementa J, et al. Somatology a Anthropology. Prague: $1^{\text {st }}$ edit State education publishing, ISBN 14-406-81, 1981.

16. Lamb SE, Ferrucci L, Volapto S, et al. Risk factors for falling in home-dwelling older women with stroke: the women's health and aging study. Stroke 2003; 34:494-501.

17. Martin R, Knubmann R, Vergal GF. Anthropologie. Stuttgart: Gustav Fischer Verlag, ISBN 3-437-30505-0, 1998.

18. Mattacola GC, Lebsack DA, Perrin HD. Intertester reliability of assessing postural sway using the Chattecx Balance System. Jur.of Athletic Training 1995; 30:237-242.

19. Pavlik J. Body stucture as an indicator of sportsman efficiency. At the link: http://home.pf.jcu.cz/ ${ }^{\sim}$ rvobr/somatotyp.htm [online], [cit. 2006-05-01].

20. Riegerova J, Ulbrichova M. Aplication of physical anthropology in the physical training and sport. Olomouc:1st. edit. Palacky University Publishing, ISBN 80-7067-847-X, 1998.

21. Waxman SG. Clinical Neuroanatomy. Lange: 25 .th edition McGraw-Hill, ISBN 7-117-05406-9, 2003.

22. Weight Bearing Squat Protocol-source on internet. [cited Oct 2006; 1 webside]. Available from: www.onbalance.com/neurocom/ protocols/motorImpairment/wbs.aspx 